\title{
Urethral Pain
}

National Cancer Institute

\section{Source}

National Cancer Institute. Urethral Pain. NCI Thesaurus. Code C78672.

A painful sensation in the urethra. 